Check for updates

Cite this: Mater. Adv., 2021, 2, 7691

Received 2nd July 2021, Accepted 13th September 2021

DOI: $10.1039 / \mathrm{d} 1 \mathrm{ma} 00572 \mathrm{c}$

rsc.li/materials-advances

\title{
Insights into the structure and ionic transport in 'water-in-bisalt' electrolytes for lithium-ion batteries $\dagger$
}

\author{
Thejus R. Kartha, Dhileep N. Reddy and Bhabani S. Mallik (D)*
}

\begin{abstract}
We investigated the dynamics and transport of lithium-ion water-in-bisalt (WiBS) electrolytes that contain lithium bis(trifluorosulfonimide) (LiTFSI) and lithium triflate (LiOTf) at different concentrations and temperatures using classical molecular dynamics simulations. The presented results are helpful to access the advantages of their use as electrolytes in Li-ion batteries. The structural analysis shows that the $\mathrm{Li}$-ions have analogous interactions with the oxygen atoms of TFSI, OTf- and water. The calculated ionic conductivities from the current autocorrelation function are close to the experimental data for all the concentrations except the lowest one, in which case, the correlated conductivity is close to the experiment. However, the Nernst-Einstein route for calculating ionic conductivity overestimates the values by a reasonably high margin. Ion-cage correlation dynamics show increasing ion-cage lifetimes with increasing concentration, which indicates that the $\mathrm{Li}$-ions remain within the anionic cages for a longer time at higher concentrations. At higher temperatures, this lifetime is seen to decrease. The statistics of non-coordinated Li-ions that are free from TFSI- and OTf- support the ion-cage dynamics.
\end{abstract}

\section{Introduction}

Li-ion batteries ${ }^{1}$ have been enjoying the prime limelight of energy research ever since they entered the market in $1991 .^{2,3}$ Since then, there have been many exciting formulations of them, and research has been progressively oriented to enhance the efficiencies of these batteries. ${ }^{4-6}$ The cathodes of these batteries are typically fabricated from oxides of lithium manganese, lithium cobaltate, $\mathrm{V}_{2} \mathrm{O}_{5}, \mathrm{FeS}_{2}$, or lithium nickel cobalt manganese and the anodes are made out of metallic lithium, graphitic or hard carbon. ${ }^{7-13} \mathrm{~A}$ Na-super-ionicconductor-structured $\mathrm{Na}_{3} \mathrm{~V}_{2}\left(\mathrm{PO}_{4}\right)_{3} / \mathrm{C}$ composite was also reported as a cathode electrode for rechargeable batteries. ${ }^{14,15}$ Electrolytes form an essential part of the setup of a battery and are responsible for the energy transfer that occurs between the electrodes. Many electrolytes like organic liquid, solid-state or polymer varieties have been devised for use in Li-ion batteries. ${ }^{16-20}$ However, the most popular and state-of-the-art batteries involve using organic carbonate solvents, and $\mathrm{LiPF}_{6}$ as the salt, which is shown to be effective in the market but is not by any means a completely safe option. ${ }^{21}$ This mixture has temperature dependencies that make it hazardous at temperatures

Department of Chemistry, Indian Institute of Technology Hyderabad, Sangareddy 502285, Telangana, India. E-mail: bhabani@chy.iith.ac.in; Tel: +91 4023016258 $\dagger$ Electronic supplementary information (ESI) available. See DOI: 10.1039/ d1ma00572c as low as $60{ }^{\circ} \mathrm{C}$, and inefficient at temperatures lower than room temperature due to the high freezing point of carbonates..$^{22,23}$ Fire accidents have occurred during the use and production of these carbonate-based Li-ion batteries. This can be attributed to their low flash points and intolerance to omnipresent entities like moisture. ${ }^{4,24,25}$ Lithium salts like $\mathrm{LiPF}_{6}$, which is the most commonly used, tend to form hydrofluoric acid within the battery casing in the presence of water, which can be corrosive. ${ }^{26,27}$ Lithium salts also have low solubility in organic solvents. To dissolve these salts, one requires a solvent with a high dielectric constant. But, with a large dielectric constant, the solvent is likely to be highly viscous. The high viscosity of the electrolyte will diminish the ion transport and hence prove to be unsuitable. ${ }^{28}$

Acknowledging these drawbacks, efforts to make safer, fireresistant and eco-friendly batteries have been carried out. Incorporating an additive like tetramethylsulfone with flameretardant properties can serve the purpose. Many reports in the literature speak about the performance of electrolytes with additives. ${ }^{29-32}$ Other alternative ways to make safer batteries demand a reformulation of the existing trend of making electrolytes. The new era of liquid electrolyte research for Liion batteries focuses on water usage as the primary solvent within a very high concentrated solution of lithium salt-waterin-salt electrolytes (WiSE). ${ }^{33}$ Water, because of its narrow electrochemical window, was deemed unfit for usage in environments of high energy density, like inside Li-ion batteries, as it would dissociate. But by super-saturating water 
with LiTFSI, the electrochemical window of water can be expanded to $\sim 3.0 \mathrm{~V}$, making it compatible with the various anodes and cathodes. This research direction also granted the research community with a Na-ion variant ${ }^{34-36}$ of WiSE and expanded its application to $4.0 \mathrm{~V}$ processes ${ }^{37}$ and higher. Once the lithium salt reaches its solubility limit, another lithium salt can be successfully dissolved to enhance the electrochemical window of water further. These are termed "Water-in-Bisalt" $(\text { WiBS })^{38}$ electrolytes. WiBS electrolytes became popular as those having an extremely high concentration of ions and the most expanded electrochemical windows for water. There has been experimental work that reports the physicochemical properties of WiBS as well. ${ }^{39,40}$ Studies on these aqueous electrolytes have provided valuable insights into many aspects of these electrolytes, and molecular dynamics simulations have served much in this respect. $^{41-44}$

This current investigation focuses on understanding the structural and transport properties of a water-in-bisalt electrolyte (LiTFSI and LiOTf) using classical molecular dynamics simulations. As suggested in previous literature, ${ }^{39}$ we use a third of the concentration of LiOTf as that of LiTFSI. We consider four concentration regimes: $5.1 \mathrm{~m}$ LiTFSI and $1.7 \mathrm{~m}$ LiOTf, $9 \mathrm{~m}$ LiTFSI and $3 \mathrm{~m}$ LiOTf, $15 \mathrm{~m}$ LiTFSI and $5 \mathrm{~m}$ LiOTf and $21 \mathrm{~m}$ LiTFSI and $7 \mathrm{~m}$ LiOTf. We also conduct a temperature-variable study on the mixture of $9 \mathrm{~m}$ LiTFSI and $3 \mathrm{~m}$ LiOTf. The calculated transport properties are compared with the available experimental data.

\section{Computational methodology}

Molecular dynamics simulations were performed using GROMACS version 5.1.2. ${ }^{45-50}$ The molecular structures used in this study have been parameterized using the Generalized Amber Force Field (GAFF), ${ }^{51}$ with the help of the Antechamber ${ }^{52}$ package. Before the molecular dynamics simulations, the individual entities used in this study were subjected to geometry optimization through electronic structure methods using the B3LYP ${ }^{53-56}$ functional with a $6-311+(2 d, p)$ basis set and RESP model ${ }^{57,58}$ to predict the partial atomic charges. The Gaussian $09^{59}$ package was used for this. In accordance with previous studies, ${ }^{60-62}$ a charge scaling factor of 0.8 was used for $\mathrm{Li}+$, TFSI- and OTf-. SPC/ $\mathrm{E}^{63}$ was the water model used. The complete list of force field parameters used in this study is provided in the ESI. $\dagger$ The optimized structures used in this study are given in Fig. 1. Following this, the Packmol ${ }^{64}$ package was used to randomly arrange the 1000 units in a cubic space. The numbers of different units to represent different concentrations used in this study are explained in Table S1 of the ESI. $\dagger$ We produced three randomly arranged initial configurations of sets of the same concentration to continue with our molecular dynamics simulations for better statistical averaging of transport properties and standard deviation calculation. The protocol followed in our work began by minimizing the energy of the initial configurations through the steepest descent algorithm. After that, each of the three systems was annealed

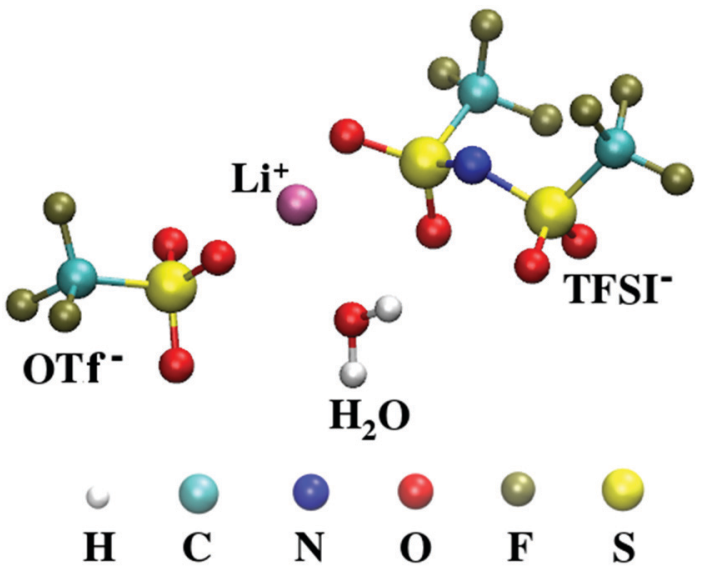

Fig. 1 The structures of all the chemical entities in this study.

to different temperatures, namely 498.15 , 698.15 and $1098.15 \mathrm{~K}$, by which slow cooling to $298.15 \mathrm{~K}$ was done with $20 \mathrm{~K}$ temperature drops at every $200 \mathrm{ps}$. They were provided with an additional $1 \mathrm{~ns}$ of equilibration concerning the $N V T$ ensemble with a reference temperature of $298.15 \mathrm{~K}$ for all the simulations. Following this, we performed equilibrations concerning the NPT and NVT ensembles, in that order, for $50 \mathrm{~ns}$ on each system. The averaged box length (5-50 ns) after the NPT equilibration was used in the input for the $N V T$ equilibration. This ensured that the average density was maintained correctly throughout the simulation. For the temperature and pressure control, the V-rescale ${ }^{65}$ thermostat and the Berendsen ${ }^{66}$ barostat algorithms were used, respectively. The production run was carried out within the NVE ensemble for $250 \mathrm{~ns}$ at an output writing frequency of $10 \mathrm{ps}$. The simulations for aqueous $15 \mathrm{~m}$ LiTFSI-5 $\mathrm{m}$ LiOTf and $21 \mathrm{~m}$ LiTFSI-7 m LiOTf were extended to $500 \mathrm{~ns}$ to facilitate calculations of correlated ionic conductivity. The convergence of physical properties after these simulations (density during the NPT equilibrations, the temperature during the $N V T$ equilibrations and total energy and temperature during the $N V E$ production run) is presented in Fig. S1 of the ESI $\dagger$ document. The linear and converging trends of these graphs show that the systems used for this study are well equilibrated. For calculations of the current autocorrelation function, ten $N V E$ simulations were produced for a period of $100 \mathrm{ps}$ each for each system in this study, with the trajectory and velocities saved every 2 fs after the final $N V E$ simulations. $N V E$ simulations for a further 4 ns were carried out in each case to calculate the ion-cage lifetimes and hydrogen bond dynamics, with an output writing frequency of 1 ps.

\section{Results and discussion}

\section{Density \& structure of WiBS electrolytes}

The densities of the electrolytes are shown in Fig. S1 (ESI $\dagger$ ) concerning different concentrations and temperatures. The increase in concentration increases the density due to a more significant number of ions in the system. But as the temperature increases, density decreases due to occupying a larger volume. 
$5.1 \mathrm{~m}$ LiTFSI, $1.7 \mathrm{~m}$ LiOTf

(a)

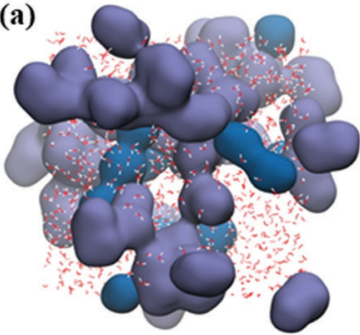

9 m LiTFSI, 3 m LiOTf

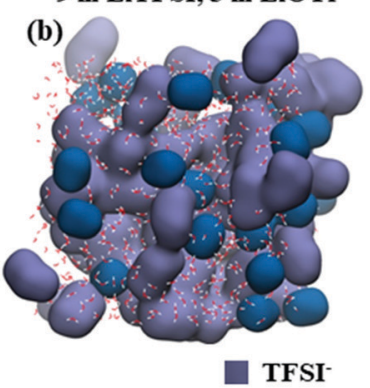

$15 \mathrm{~m}$ LiTFSI, $5 \mathrm{~m}$ LiOTf

(c)

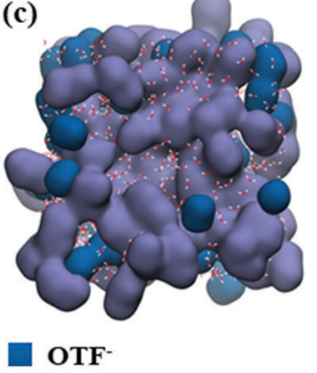

$21 \mathrm{~m}$ LiTFSI, 7 m LiOTf

(d)

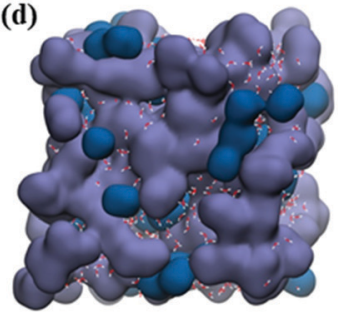

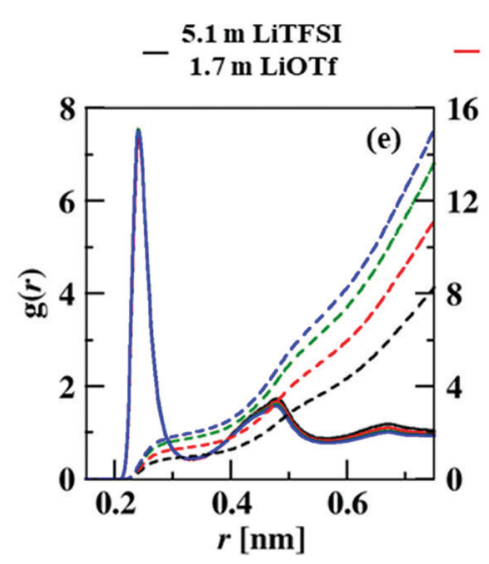
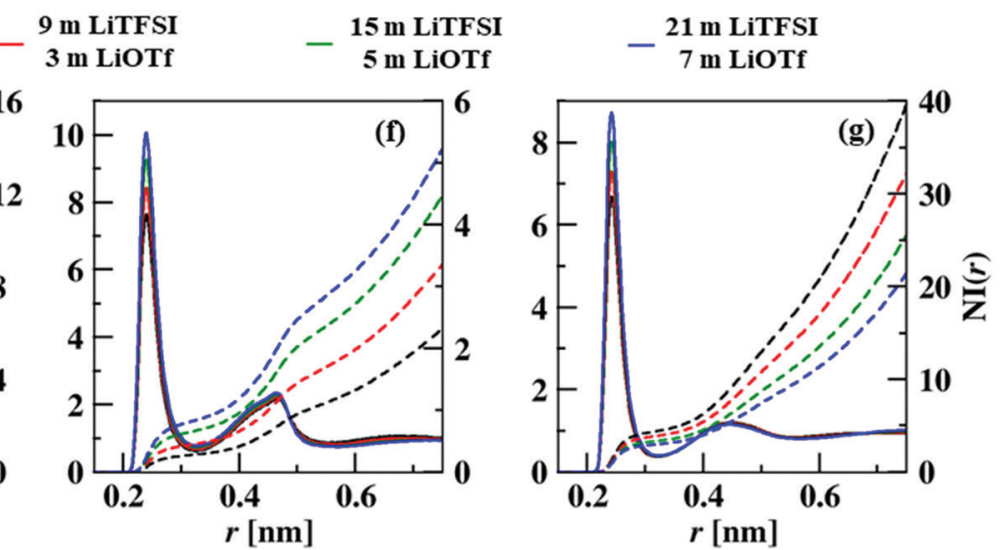

Fig. 2 (a-d) Snapshots of the water-in-bisalt system at different concentrations. RDF of the $x \mathrm{~m} \mathrm{LiTFSI}$ and $x / 3 \mathrm{~m}$ LiOTf aqueous mixtures: (e) Li ${ }^{+}-\mathrm{O}$ of $\mathrm{TFSI}^{-}$, (f) $\mathrm{Li}^{+}-\mathrm{O}$ of $\mathrm{OTf}^{-}$and (g) $\mathrm{Li}^{+}-\mathrm{O}$ of water. Anions are presented as large volumes; $\mathrm{Li}+$ and water are shown with atomistic details. The dashed lines represent the coordination numbers at various lengths from the reference atoms.

The thorough analysis of trajectories provides a distinction between the regions of ionic moieties of the system. The snapshots that are portraying various structures are presented in Fig. 2(a-d). As the concentration increases, the volume occupied by the surfaces representing TFSI $^{-}$and OTf increases. The water molecules move between these anionic regions, like 'water channels, ${ }^{43}$ which are assumed to be present in concentrated aqueous electrolytes of batteries. This phenomenon was also reported earlier as 'percolation' based on graph theory related to molecular aggregation in aqueous solutions. ${ }^{67}$ Since the anions contain oxygen atoms that can form hydrogen bonds with the water molecules, one can expect a strong hydrogen bonding network in these electrolyte systems. These dynamics will be investigated more thoroughly in the later sections.

For more quantitative information of the ionic structuring in this system, radial distribution functions (RDFs) were calculated between $\mathrm{Li}^{+}$and oxygen atoms of $\mathrm{TFSI}^{-}$, OTf ${ }^{-}$and water, given in Fig. 2(e-g). Interestingly, all three of these correlations show similar ranges of interaction in their first solvation shells, i.e., the first solvation shell peak remains unchanged mainly in terms of its height, even though the concentrations of lithium salts change drastically in each case. The position of these peaks also does not alter in any of the three interactions. The first solvation shell begins at a little beyond $0.20 \mathrm{~nm}$ and extends till $\sim 0.33 \mathrm{~nm}$ in all cases. The preliminary indication of these observations is that the solvation structure does not significantly change when the concentration is altered. TFSI does not change its solvation characteristics towards $\mathrm{Li}^{+}$even with significant changes in the overall concentration of this system, according to the RDF data, as represented in Fig. 2(e).

\section{Ionic conductivity}

To study the transport of ions in these electrolytes, we calculated the mean squared displacement (MSD) of each ion in the system using the following formula:

$$
\operatorname{MSD}=\frac{1}{N} \sum_{i=1}^{N}\left[r_{i}(t)-r_{i}(0)\right]^{2}
$$

Where $N$ is the total number of particles in the system and $r_{i}(t)$ is the position of the ith particle at time $t$. The corresponding $\log -\log$ plot of MSD and the $\beta(t)$ vs. $t$ plot $^{68}$ helps decide the electrolyte system's diffusive regime. $\beta(t)$ is defined as:

$$
\beta(t)=\frac{\mathrm{d} \ln (\mathrm{MSD})}{\mathrm{d} \ln (t)}
$$

Along the simulation time, the log-log plots show a straight line, and the value of $\beta(t)$ is unity, considered to be the diffusive regime of the electrolytes. The data for MSD, log-log plots and the $\beta(t)$ plots are provided in Fig. 3 .

Fig. 3(a) shows a decrease in the MSD of $\mathrm{Li}^{+}$as concentration increases, while it shows a significant increase when the temperature is raised. The same effect is observed in the anions (Fig. 3(d)), although they traverse much lesser than the $\mathrm{Li}^{+}$, 

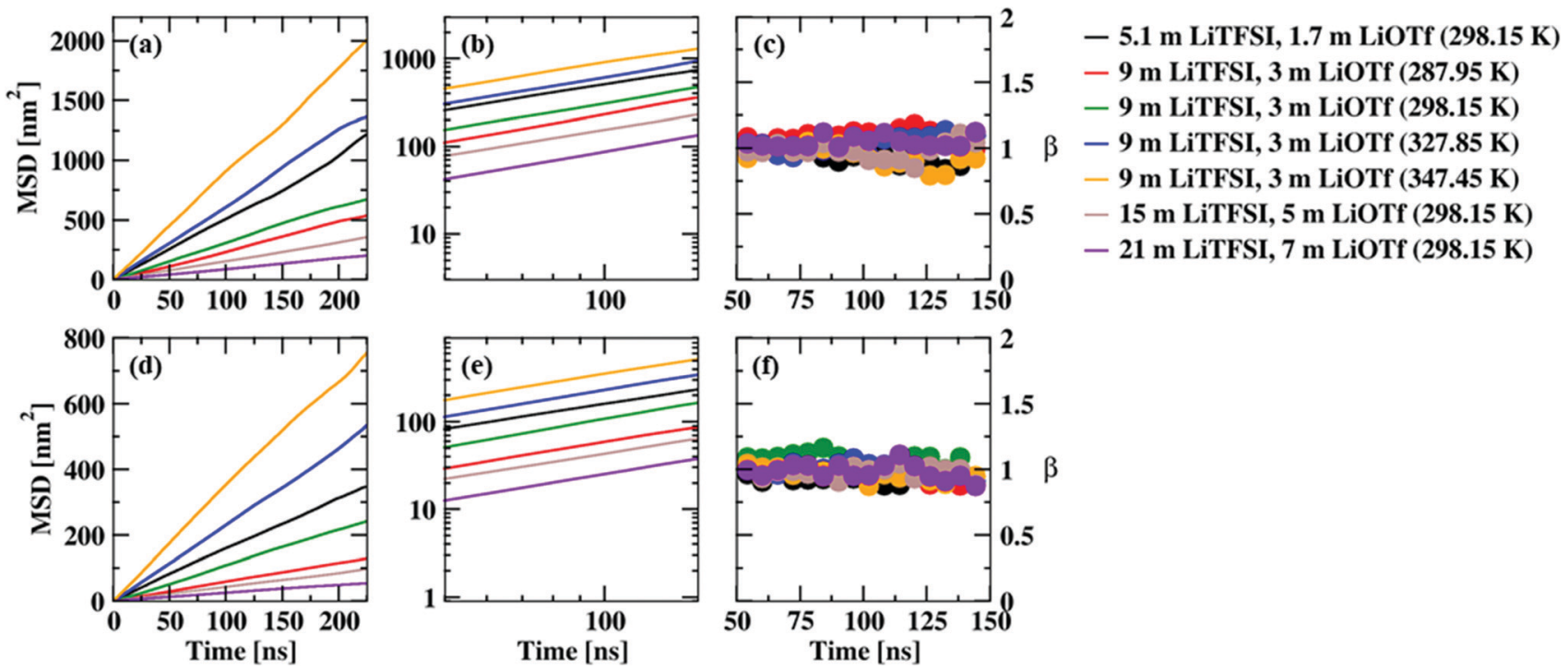

Fig. 3 (a) MSD vs. time, (b) the corresponding log-log plot and (c) $\beta$ vs. time plots of $\mathrm{Li}^{+}$and (d) MSD vs. time, (e) the corresponding log-log plot and (f) $\beta$ vs. time plots of the anions.

attributed to their larger sizes and bulkier structures. The log$\log$ plots and the $\beta(t)$ plots are represented across a time of $50-150 \mathrm{~ns}$ of the simulation. This is the region where the former shows straight-line behavior and the latter converging to a value of 1 . Hence, this is the diffusive regime for this aqueous LiTFSI-LiOTf system for all the studied concentrations and temperatures. To know the individual contributions to ionic conductivity, it is often preferred to use the Einstein relation ${ }^{69}$ to derive the self-diffusion coefficients of each type of ion present in the system by fitting it in the diffusive regime:

$$
D=\lim _{t \rightarrow \infty} \frac{1}{6 N t} \sum_{j=1}^{N}\left[r_{j}(t)-r_{j}(0)\right]^{2}
$$

where $D$ is the self-diffusion coefficient, $N$ is the total number of particles in the system, $t$ is the length of the diffusive regime and, $r_{j}(t)$ is the position of the jth particle at time $t$. From here, we apply these self-diffusion coefficients into the Nernst-Einstein ${ }^{70,71}$ relation to compute the ionic conductivity of the system:

$$
\sigma=\frac{N q_{+} q_{-}}{V k_{\mathrm{B}} T}\left(D_{+}+D_{-}\right)
$$

Here, $\sigma$ denotes the ionic conductivity, $N$ the number of ionic species in the system, $q_{+}$and $q_{-}$the cationic and anionic charges, respectively, $V$ the volume of the simulation box, $k_{\mathrm{B}}$ the Boltzmann constant, $T$ the temperature at which the simulation is run and $D_{+}$ and $D_{-}$represent the self-diffusion coefficients of the cation and anion, respectively. The ionic conductivity obtained through this route is presented in Fig. S2 (ESI $\dagger$ ). The experimental values ${ }^{39}$ are also provided for comparison. The ionic conductivity decreases with an increase in the salt concentrations due to the reduced mobility of the ions in highly concentrated electrolytes but increases with temperature. Eqn (4) provides the ionic conductivity directly by plugging in the values. Still, it is also observed to overestimate the ionic conductivity by a substantial margin (as observed in Fig. S2, ESI $\dagger$ ) since it does not consider the correlated motion of the ions. This motion has a severe impact on the actual transport that the ions take part in. To overcome this, the preferred choice is to calculate the correlated conductivity: $:^{33,72-74}$

$$
\sigma=\frac{1}{6 k_{\mathrm{B}} T V} \lim _{t \rightarrow \infty} \frac{\mathrm{d}}{\mathrm{d} t}\left\langle\sum_{i=1}^{N} \sum_{j=1}^{N} \begin{array}{c}
q_{i} q_{j}\left[\vec{r}_{i}(t)-\vec{r}_{i}(0)\right] \\
{\left[\vec{r}_{j}(t)-\vec{r}_{j}(0)\right]}
\end{array}\right\rangle .
$$

Fig. 4 shows the calculated correlated ionic conductivities of all the studied concentrations and temperatures concerning their corresponding experimental counterparts as published by Ding et $a l . .^{39}$ The proximity of our calculated values to the experimental data shows the accuracy of our model. However, we observe that the calculated values of ionic conductivity show a deviation from experimental values at high concentrations. Following the trend of the experimental data, the ionic conductivity exhibits a decreasing behavior with the increase in concentration.

Another essential method to consider for the calculation of ionic conductivity is via the integration of the current autocorrelation function (CACF), which is calculated via a Green-Kubo relation: ${ }^{72,75,76}$

$$
\sigma=\frac{1}{3 k_{\mathrm{B}} T V} \int_{0}^{+\infty}\left\langle\boldsymbol{J}\left(t_{0}+t\right) \cdot \boldsymbol{J}\left(t_{0}\right)\right\rangle \mathrm{d} t
$$

where $\boldsymbol{J}$ is the electrical current which is obtained as the product of ionic charge $(q)$ and velocity $(\boldsymbol{v})$ over $N$ species:

$$
\boldsymbol{J}=\sum_{i=1}^{N} q_{i} \boldsymbol{v}_{i}
$$

This method has been popular among many researchers and has been deployed extensively in many ionic liquids. ${ }^{77-79}$ The autocorrelation functions obtained in each case are presented in Fig. S3 and S4 (ESI $\dagger$ ). To get appropriate ionic 

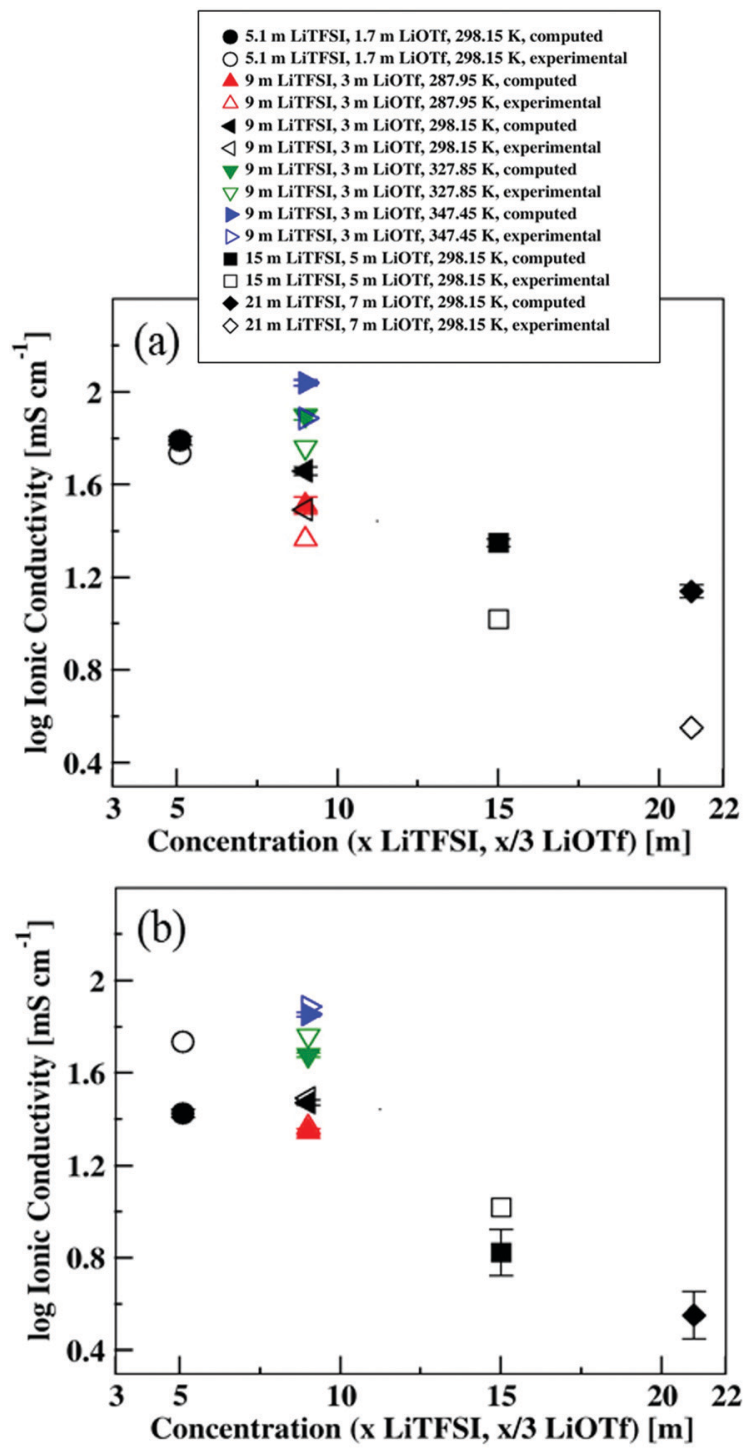

Fig. 4 lonic conductivity of the electrolytes and comparison with experimental values at different concentrations and temperatures through various methods: (a) correlated ionic conductivity, (b) ionic conductivity from the current autocorrelation function. The legends and experimental values ${ }^{37}$ have been provided for reference.

conductivity values, the Green-Kubo integral (eqn (6)) is evaluated over specific cutoff times. These values of ionic conductivity and the cutoff times used are recorded in Table S2 of the ESI. $\dagger$ The ionic conductivity obtained by this method is provided in Fig. 4(b). In this case, we observe that at a lower concentration of the electrolyte (at $5.1 \mathrm{~m}$ LiTFSI, $1.7 \mathrm{~m} \mathrm{LiOTf}$ ), the ionic conductivity is underestimated. Still, as the concentration increases, it predicts the values reasonably close to the experiment. At a concentration of $21 \mathrm{~m}$ LiTFSI and $7 \mathrm{~m}$ LiOTf, the calculated ionic conductivity values almost overlap with the experimental value.

These values are in a better agreement with the experiment across all concentrations than values calculated from either of the two methods individually, with minimal error in each case. After various analysis to find a suitable method to report the conductivities, we found that except the lowest electrolyte concentration, the values obtained from CACF match well with that of experiments. However, the calculated correlated conductivity of the lowest concentration agrees well with the experimental value. These comparative values are shown in Fig. 4. The computed values also represent the change in conductivity concerning change in concentration and temperature correctly. Our models of both methods for reporting ionic conductivities can be justified as appropriately representative of the dynamic features of water-in-bisalt electrolyte in the mentioned concentrations and temperatures. As the concentration increases, the number of ions taking part in the transport increases, but the freedom for each ion to move decreases due to the rise in the effective total of attractive and repulsive Coulombic forces experienced by each ion. But when the temperature is increased, the ionic conductivity also increases due to the higher kinetic energy of the entities. In the light of commercial requirements of ionic conductivity for a Li-ion battery, these electrolytes can be considered along with state-of-the-art electrolytes. ${ }^{80,81}$

\section{Ion-cage lifetime}

The ions in electrolytes are primarily surrounded by several counterions due to coulombic interactions; the additives disrupt the solvation structure by interacting with the ions. At a relatively high salt concentration like WiBS, the cation is trapped by anions in its first solvation shell. Due to strong coulombic interactions, the formed solvation shell appears as an ion cage structure. The trapped ions do not escape from the cage for high concentrated electrolytes and hop out with a larger time scale. The time they are trapped inside the cage can be correlated to ion cage lifetime that depends on the viscosity of the electrolyte. The ion cage structure plays a vital role in describing the overall dynamics of the electrolytes due to correlated interactions of counter ions. The dynamics of the escape process can be explained by investigating the time correlation function of the formation of cage structure. The method is like Luzar and Chandler's method ${ }^{82,83}$ on hydrogenbond dynamics in liquid water ${ }^{84-86}$ that paved the way to significant insights into lifetimes and other vital properties of hydrogen bonds. The concept also was expanded into ionic liquid and other nonaqueous cases in the more recent past. ${ }^{85-88}$ There have been two broad classifications while looking at the lifetimes of hydrogen bonds - continuous ${ }^{89}$ and intermittent lifetime. ${ }^{90}$ This idea can be generalized to any system, where there is a dynamic interaction between two moieties, and when there is an ionic entity solvated by other ionic groups forming an ion-cage. ${ }^{68,91,92}$ The solvation of ionic entities can be viewed from the perspective of ionic cages. To understand the ionic dynamics, we calculated the ion-cage lifetimes, according to the equation: ${ }^{89,93-96}$

$$
C(t) \simeq \frac{\langle h(0) h(t)\rangle}{\langle h\rangle}
$$

The function $h(t)$ is dependent on a cutoff radius - it is treated to be unity if ionic groups of interest are within a 

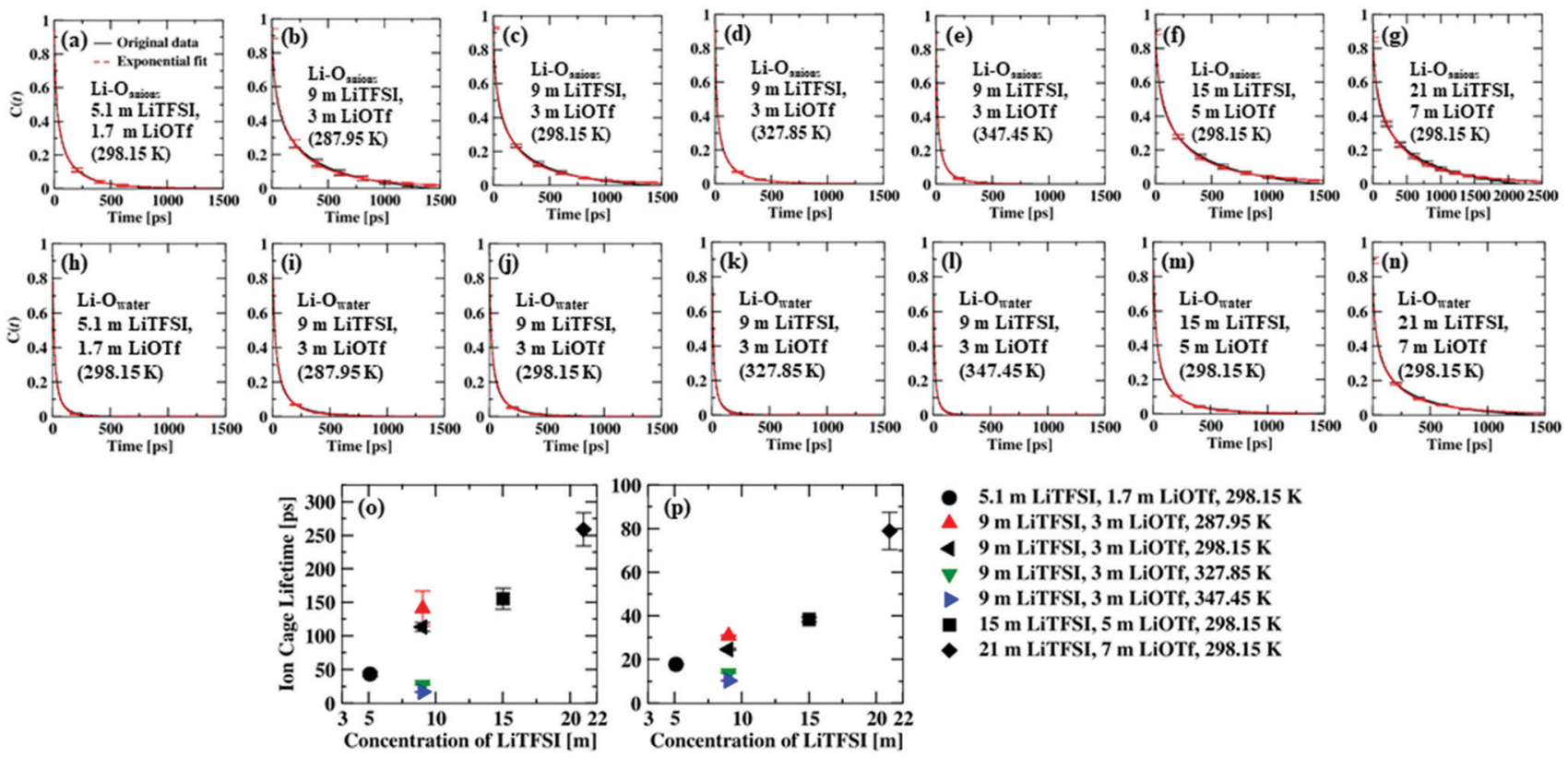

- $5.1 \mathrm{~m}$ LiTFSI, $1.7 \mathrm{~m}$ LiOTf, $298.15 \mathrm{~K}$

$9 \mathrm{~m} \mathrm{LiTFSI}, 3 \mathrm{~m}$ LiOTf, $287.95 \mathrm{~K}$

$9 \mathrm{~m} \mathrm{LiTFSI,} 3 \mathrm{~m} \mathrm{LiOTf}, 298.15 \mathrm{~K}$

$79 \mathrm{~m} \mathrm{LiTFSI}, 3 \mathrm{~m} \mathrm{LiOTf}, 327.85 \mathrm{~K}$

$9 \mathrm{~m}$ LiTFSI, $3 \mathrm{~m}$ LiOTf, $347.45 \mathrm{~K}$

- $15 \mathrm{~m}$ LiTFSI, $5 \mathrm{~m}$ LiOTf, $298.15 \mathrm{~K}$

- 21 m LiTFSI, 7 m LiOTf, $298.15 \mathrm{~K}$

Fig. 5 Ion cage correlation functions and fitted curves for $(a-g) \mathrm{Li}-\mathrm{O}$ of anion and $(\mathrm{h}-\mathrm{n}) \mathrm{Li}-\mathrm{O}$ of water at different concentrations; ion cage lifetimes of (o) $\mathrm{Li}-\mathrm{O}$ of anion and (p) $\mathrm{Li}-\mathrm{O}$ of water at different concentrations and temperatures.

particular cutoff distance to each other. Otherwise, it is zero. The autocorrelation function $C(t)$ provides the probability of $\mathrm{Li}^{+}$ and an anion that remain in the same cage until time $t$. According to the RDF presented in the earlier section of this manuscript, we understand that the first solvation shells do not extend beyond $0.33 \mathrm{~nm}$. This distance is taken as the cutoff distance for this calculation. The decay of the autocorrelation function can be quantified by calculating the concerned time scale of the decay. Eqn (8) results in an autocorrelation function that has a decaying character, and which is then fitted with a stretched exponential function ${ }^{97}$ as shown in eqn (9):

$$
y=a_{0} \exp \left(\frac{-x}{\tau_{1}}\right)^{\tau_{2}}
$$

Here, $\tau_{1}$ is ion-cage lifetime and $\tau_{2}$ is the stretching parameter. For the interaction of $\mathrm{Li}^{+}$with the oxygen atoms of TFSI and OTf $^{-}$, the plots corresponding to eqn (8) and their corresponding fits with eqn (9) are provided in Fig. $5(\mathrm{a}-\mathrm{g})$ for varying concentrations and temperatures of the electrolyte. The autocorrelation functions show good fitting concerning the stretched exponential function. The complete list of fitting parameters used here has been tabulated in the Table S3 (ESI $\dagger$ ). Similarly, for the interaction of $\mathrm{Li}^{+}$with the oxygen atoms of water, curves fitted with the stretched exponential function are shown in Fig. 5(h-n). The fitting parameters used for this are provided in Table S4 (ESI $\dagger$ ). The ion-cage lifetimes derived from the fitting are consolidated in Fig. 5(o-p).

In both $\mathrm{Li}-\mathrm{O}_{\text {anions }}$ and $\mathrm{Li}-\mathrm{O}_{\text {water }}$ cases, we observe an increase in ion-cage lifetime values when the concentration increases. This is because of an increased number of anions that make it difficult for the $\mathrm{Li}^{+}$to leave their vicinity and stay in the vicinity for a more extended period. However, when the temperature increases, there is a decrease in the ion-cage lifetime, majorly because of the faster motion of the $\mathrm{Li}^{+}$ions at higher temperatures. The rise in temperature increases the dynamics of the $\mathrm{Li}^{+}$, and they tend to leave the ion-cage faster. The increasing MSD of the ions with increasing temperature complements this. Comparing Fig. 5(o and p), we observe that, even though the same trend is followed by ion-cage lifetimes, there is a significant difference in the values. For a concen-

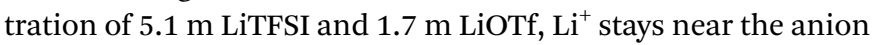
oxygen atoms for an average of $43.7 \mathrm{ps}$. But, in the case of oxygen molecules of water around the $\mathrm{Li}^{+}$, the average lifetime is 17.8 ps. At higher concentrations, this effect is more pronounced. In the electrolyte system containing $21 \mathrm{~m}$ LiTFSI and $7 \mathrm{~m}$ LiOTf, the $\mathrm{Li}-\mathrm{O}_{\text {anions }}$ lifetime shows an average of $259.0 \mathrm{ps}$, while for $\mathrm{Li}-\mathrm{O}_{\text {water }}$, it is only $79.0 \mathrm{ps}$. Ion-cage lifetimes are correlated to the ionic conductivity of the electrolytes. With increase in ion-cage lifetime, the counter ions stay close to each other, which affects the overall conductivity.

\section{Conclusions}

In this era where most of the research is focused on developing and advancing energy storage systems, the efficiency of electrolytes becomes essential. The new family of aqueous electrolytes has been in vogue as far as electrolyte research is concerned. We modeled the LiTFSI - LiOTf WiBS electrolyte system for various concentrations and temperatures and investigated their workings from a molecular dynamics perspective. Calculation of RDF shows that the interaction strength of $\mathrm{Li}^{+}$with the oxygen atoms of $\mathrm{TFSI}^{-}$, OTf ${ }^{-}$and water are very similar, with minor changes in $\mathrm{Li}^{+}-\mathrm{O}$ of $\mathrm{OTf}^{-}$and $\mathrm{Li}^{+}-\mathrm{O}$ of water as the concentration increases. Our finding is in accord with the 
earlier reported simulation study. ${ }^{98}$ The calculation of mean squared displacement and the choice of an appropriate diffusive regime helped calculate the ionic conductivity of these electrolyte systems. The use of the Nernst-Einstein relation to calculate ionic conductivity showed a significant overestimation of values, while correlated conductivity calculations yielded values reasonably close to experimentally obtained ones. The ionic conductivity increases with temperature but decreases with an increase in concentration. The ions experience difficulty in free motion due to a higher number of attractive and repulsive forces. However, all these ionic conductivity values were found to satisfy commercial requirements and were higher than that of the state-of-the-art battery electrolytes. The ion-cage correlation functions calculated and fitted with a stretched exponential function helped derive the ion-cage lifetimes of the different concentrations of WiBS electrolytes and at different temperatures. These values showed an increase with increasing concentration and decreased with an increase in temperature. Through appropriate, the electrolytes have not been adopted in large scale commercial purposes. Further studies will be required to find the effects of change in thermodynamic conditions on the formation of solid and cathode electrolyte interfaces. The involved unwanted chemical reactions can enlighten the disadvantages of these electrolytes. Our results help us understand that these WiBS electrolyte systems are efficient in ion transport and have intriguing structural features. These analyses will help understand the microstructure of these electrolyte systems and help further the development of the same.

\section{Conflicts of interest}

There are no conflicts to declare.

\section{Acknowledgements}

The financial support (DST/NSM/R\&D_HPC_Applications/Sanction/ 2021/06) for this work was provided by the National Supercomputing Mission (NSM), India.

\section{References}

1 M. S. Whittingham, Electrical Energy Storage and Intercalation Chemistry, Science, 1976, 192, 1126-1127.

2 G. Eichinger and J. O. Besenhard, High energy density lithium cells: Part II. Cathodes and complete cells, J. Electroanal. Chem. Interfacial Electrochem., 1976, 72(1), 1-31.

3 K. Mizushima, P. C. Jones, P. J. Wiseman and J. B. Goodenough, $\operatorname{LixCoO}_{2}(0<\mathrm{x} \leq 1)$ : A new cathode material for batteries of high energy density, Solid State Ionics, 1981, 3-4, 171-174.

$4 \mathrm{~K} . \mathrm{Xu}$, Nonaqueous Liquid Electrolytes for Lithium-Based Rechargeable Batteries, Chem. Rev., 2004, 104, 4303-4418.

5 D. Aurbach, Y. Talyosef, B. Markovsky, E. Markevich, E. Zinigrad, L. Asraf, J. S. Gnanaraj and H.-J. Kim, Design of electrolyte solutions for $\mathrm{Li}$ and Li-ion batteries: a review, Electrochim. Acta, 2004, 50, 247-254.

6 E. Peled, The Electrochemical Behavior of Alkali and Alkaline Earth Metals in Nonaqueous Battery Systems-The Solid Electrolyte Interphase Model, J. Electrochem. Soc., 1979, 126, 2047-2051.

7 D. T. Tran, H. Dong, S. D. Walck and S. S. Zhang, Pyrite FeS2-C composite as a high capacity cathode material of rechargeable lithium batteries, $R S C A d v$., 2015, 5, 87847-87854.

8 J. Shu, M. Shui, F. Huang, Y. Ren, Q. Wang, D. Xu and L. Hou, A New Look at Lithium Cobalt Oxide in a Broad Voltage Range for Lithium-Ion Batteries, J. Phys. Chem. C, 2010, 114, 3323-3328.

9 W. Xu, J. Wang, F. Ding, X. Chen, E. Nasybulin, Y. Zhang and J.-G. Zhang, Lithium metal anodes for rechargeable batteries, Energy Environ. Sci., 2014, 7, 513-537.

10 N. Nitta, F. Wu, J. T. Lee and G. Yushin, Li-ion battery materials: present and future, Mater. Today, 2015, 18, 252-264.

11 H. Zheng, Q. Qu, L. Zhang, G. Liu and V. S. Battaglia, Hard carbon: a promising lithium-ion battery anode for high temperature applications with ionic electrolyte, RSC Adv., 2012, 2, 4904-4912.

12 Y. Yang, L. Li, H. Fei, Z. Peng, G. Ruan and J. M. Tour, Graphene Nanoribbon/V2O5 Cathodes in Lithium-Ion Batteries, ACS Appl. Mater. Interfaces, 2014, 6, 9590-9594.

13 Y. Kim, Lithium Nickel Cobalt Manganese Oxide Synthesized Using Alkali Chloride Flux: Morphology and Performance As a Cathode Material for Lithium Ion Batteries, ACS Appl. Mater. Interfaces, 2012, 4, 2329-2333.

14 W. Song, X. Cao, Z. Wu, J. Chen, K. Huangfu, X. Wang, Y. Huang and X. Ji, A study into the extracted ion number for NASICON structured Na3V2(PO4)3 in sodium-ion batteries, Phys. Chem. Chem. Phys., 2014, 16, 17681-17687.

15 X. Zhang, J. Ma, P. Hu, B. Chen, C. Lu, X. Zhou, P. Han, L. Chen and G. Cui, An insight into failure mechanism of NASICON-structured Na3V2(PO4)3 in hybrid aqueous rechargeable battery, J. Energy Chem., 2019, 32, 1-7.

16 S. Seki, N. Serizawa, K. Takei, K. Dokko and M. Watanabe, Charge/discharge performances of glyme-lithium salt equimolar complex electrolyte for lithium secondary batteries, J. Power Sources, 2013, 243, 323-327.

17 M. Nojabaee, J. Popovic and J. Maier, Glyme-based liquidsolid electrolytes for lithium metal batteries, J. Mater. Chem. A, 2019, 7, 13331-13338.

$18 \mathrm{~K}$. Xu, Tailoring Electrolyte Composition for LiBOB, J. Electrochem. Soc., 2008, 155, A733-A738.

19 L. Long, S. Wang, M. Xiao and Y. Meng, Polymer electrolytes for lithium polymer batteries, J. Mater. Chem. A, 2016, 4, 10038-10069.

20 W. Zhao, J. Yi, P. He and H. Zhou, Solid-State Electrolytes for Lithium-Ion Batteries: Fundamentals, Challenges and Perspectives, Electrochem. Energy Rev., 2019, 2, 574-605.

21 M. Li, C. Wang, Z. Chen, K. Xu and J. Lu, New Concepts in Electrolytes, Chem. Rev., 2020, 120, 6783-6819. 
22 A. Mauger and C. M. Julien, Critical review on lithium-ion batteries: are they safe? Sustainable?, Ionics, 2017, 23, 1933-1947.

23 A. Manthiram, An Outlook on Lithium Ion Battery Technology, ACS Cent. Sci., 2017, 3, 1063-1069.

24 J. B. Goodenough and Y. Kim, Challenges for Rechargeable Li Batteries, Chem. Mater., 2010, 22, 587-603.

25 J.-M. Tarascon and M. Armand, Issues and challenges facing rechargeable lithium batteries, Nature, 2001, 414, 359.

26 S. F. Lux, J. Chevalier, I. T. Lucas and R. Kostecki, HF Formation in LiPF6-Based Organic Carbonate Electrolytes, ECS Electrochem. Lett., 2013, 2, A121.

27 Z. Chen, N. Xu, W. Li, R. Zhao, Y. Dong, J. Liu, C. Su, J. Wang and C. Zhang, Effect of trace hydrofluoric acid in a LiPF6 electrolyte on the performance of a Li-organic battery with an N-heterocycle based conjugated microporous polymer as the cathode, J. Mater. Chem. A, 2019, 7, 16347-16355.

28 S. Han, Structure and dynamics in the lithium solvation shell of nonaqueous electrolytes, Sci. Rep., 2019, 9, 1-10.

$29 \mathrm{~K} . \mathrm{Xu}$ and C. A. Angell, Sulfone-Based Electrolytes for Lithium-Ion Batteries, J. Electrochem. Soc., 2002, 149, A920-A926.

30 G. Kumar, T. R. Kartha and B. S. Mallik, Novelty of Lithium Salt Solution in Sulfone and Dimethyl Carbonate-Based Electrolytes for Lithium-Ion Batteries: A Classical Molecular Dynamics Simulation Study of Optimal Ion Diffusion, J. Phys. Chem. C, 2018, 122, 26315-26325.

31 T. Aashish and B. S. Mallik, Rattling Transport of Lithium Ion in the Cavities of Model Solid Electrolyte Interphase, J. Phys. Chem. C, 2019, 123, 25015-25024.

32 T. R. Kartha and B. S. Mallik, Revisiting LiClO4 as an electrolyte for Li-ion battery: Effect of aggregation behavior on ion-pairing dynamics and conductance, J. Mol. Liq., 2020, 302, 112536.

33 L. Suo, O. Borodin, T. Gao, M. Olguin, J. Ho, X. Fan, C. Luo, C. Wang and K. Xu, "Water-in-salt" electrolyte enables highvoltage aqueous lithium-ion chemistries, Science, 2015, 350, 938-943.

34 R.-S. Kühnel, D. Reber and C. Battaglia, A High-Voltage Aqueous Electrolyte for Sodium-Ion Batteries, ACS Energy Lett., 2017, 2, 2005-2006.

35 J. Han, H. Zhang, A. Varzi and S. Passerini, FluorineFree Water-in-Salt Electrolyte for Green and Low-Cost Aqueous Sodium-Ion Batteries, ChemSusChem, 2018, 11, 3704-3707.

36 L. Suo, O. Borodin, Y. Wang, X. Rong, W. Sun, X. Fan, S. Xu, M. A. Schroeder, A. V. Cresce, F. Wang, C. Yang, Y.-S. Hu, $\mathrm{K}$. $\mathrm{Xu}$ and C. Wang, "Water-in-Salt" Electrolyte Makes Aqueous Sodium-Ion Battery Safe, Green, and LongLasting, Adv. Energy Mater., 2017, 7, 1701189.

37 C. Yang, J. Chen, T. Qing, X. Fan, W. Sun, A. von Cresce, M. S. Ding, O. Borodin, J. Vatamanu, M. A. Schroeder, N. Eidson, C. Wang and K. Xu, 4.0 V Aqueous Li-Ion Batteries, Joule, 2017, 1, 122-132.

38 L. Suo, O. Borodin, W. Sun, X. Fan, C. Yang, F. Wang, T. Gao, Z. Ma, M. Schroeder, A. von Cresce, S. M. Russell,
M. Armand, A. Angell, K. Xu and C. Wang, Advanced HighVoltage Aqueous Lithium-Ion Battery Enabled by "Water-inBisalt" Electrolyte, Angew. Chem., Int. Ed., 2016, 55, 7136-7141.

39 M. S. Ding, A. von Cresce and K. Xu, Conductivity, Viscosity, and Their Correlation of a Super-Concentrated Aqueous Electrolyte, J. Phys. Chem. C, 2017, 121, 2149-2153.

40 J. Forero-Saboya, E. Hosseini-Bab-Anari, M. E. Abdelhamid, K. Moth-Poulsen and P. Johansson, Water-in-Bisalt Electrolyte with Record Salt Concentration and Widened Electrochemical Stability Window, J. Phys. Chem. Lett., 2019, 10, 4942-4946.

41 Z. Li, R. Bouchal, T. Mendez-Morales, A.-L. Rollet, C. Rizzi, S. Le Vot, F. Favier, B. Rotenberg, O. Borodin, O. Fontaine and M. Salanne, Transport Properties of Li-TFSI Water-inSalt Electrolytes, J. Phys. Chem. B, 2019, 123, 10514-10521.

42 O. Borodin, L. Suo, M. Gobet, X. Ren, F. Wang, A. Faraone, J. Peng, M. Olguin, M. Schroeder, M. S. Ding, E. Gobrogge, A. von Wald Cresce, S. Munoz, J. A. Dura, S. Greenbaum, C. Wang and K. Xu, Liquid Structure with NanoHeterogeneity Promotes Cationic Transport in Concentrated Electrolytes, ACS Nano, 2017, 11, 10462-10471.

43 J. Lim, K. Park, H. Lee, J. Kim, K. Kwak and M. Cho, Nanometric Water Channels in Water-in-Salt Lithium Ion Battery Electrolyte, J. Am. Chem. Soc., 2018, 140, 15661-15667.

44 J. S. Babu and S. P. Sathian, The role of activation energy and reduced viscosity on the enhancement of water flow through carbon nanotubes, J. Chem. Phys., 2011, 134, 194509.

45 M. J. Abraham, T. Murtola, R. Schulz, S. Páll, J. C. Smith, B. Hess and E. Lindahl, GROMACS: High performance molecular simulations through multi-level parallelism from laptops to supercomputers, SoftwareX, 2015, 1-2, 19-25.

46 S. Páll, M. J. Abraham, C. Kutzner, B. Hess and E. Lindahl, in Solving Software Challenges for Exascale, ed. S. Markidis and E. Laure, Springer International Publishing, 2015, pp. 3-27.

47 H. J. C. Berendsen, D. van der Spoel and R. van Drunen, GROMACS: A message-passing parallel molecular dynamics implementation, Comput. Phys. Commun., 1995, 91, 43-56.

48 B. Hess, C. Kutzner, D. van der Spoel and E. Lindahl, GROMACS 4: Algorithms for Highly Efficient, LoadBalanced, and Scalable Molecular Simulation, J. Chem. Theory Comput., 2008, 4, 435-447.

49 S. Pronk, S. Páll, R. Schulz, P. Larsson, P. Bjelkmar, R. Apostolov, M. R. Shirts, J. C. Smith, P. M. Kasson, D. van der Spoel, B. Hess and E. Lindahl, GROMACS 4.5: a high-throughput and highly parallel open source molecular simulation toolkit, Bioinformatics, 2013, 29, 845-854.

50 E. Lindahl, B. Hess and D. van der Spoel, GROMACS 3.0: a package for molecular simulation and trajectory analysis, Mol. Model. Annu., 2001, 7, 306-317.

51 J. Wang, R. M. Wolf, J. W. Caldwell, P. A. Kollman and D. A. Case, Development and testing of a general amber force field, J. Comput. Chem., 2004, 25, 1157-1174. 
52 J. Wang, W. Wang, P. A. Kollman and D. A. Case, Automatic atom type and bond type perception in molecular mechanical calculations, J. Mol. Graphics Modell., 2006, 25, 247-260.

53 A. D. Becke, A new mixing of Hartree-Fock and local densityfunctional theories, J. Chem. Phys., 1993, 98, 1372-1377.

54 C. Lee, W. Yang and R. G. Parr, Development of the ColleSalvetti correlation-energy formula into a functional of the electron density, Phys. Rev. B: Condens. Matter Mater. Phys., 1988, 37, 785-789.

55 S. H. Vosko, L. Wilk and M. Nusair, Accurate spindependent electron liquid correlation energies for local spin density calculations: a critical analysis, Can. J. Phys., 1980, 58, 1200-1211.

56 P. J. Stephens, F. J. Devlin, C. F. Chabalowski and M. J. Frisch, Ab Initio Calculation of Vibrational Absorption and Circular Dichroism Spectra Using Density Functional Force Fields, J. Phys. Chem., 1994, 98, 11623-11627.

57 W. D. Cornell, P. Cieplak, C. I. Bayly and P. A. Kollman, Application of RESP charges to calculate conformational energies, hydrogen bond energies, and free energies of solvation, J. Am. Chem. Soc., 1993, 115, 9620-9631.

58 C. I. Bayly, P. Cieplak, W. Cornell and P. A. Kollman, A wellbehaved electrostatic potential based method using charge restraints for deriving atomic charges: the RESP model, J. Phys. Chem., 1993, 97, 10269-10280.

59 M. J. Frisch, G. W. Trucks, H. B. Schlegel, G. E. Scuseria, M. A. Robb, J. R. Cheeseman, G. Scalmani, V. Barone, G. A. Petersson, H. Nakatsuji, X. Li, M. Caricato, A. Marenich, J. Bloino, B. G. Janesko, R. Gomperts, B. Mennucci, H. P. Hratchian, J. V. Ortiz, A. F. Izmaylov, J. L. Sonnenberg, D. Williams-Young, F. Ding, F. Lipparini, F. Egidi, J. Goings, B. Peng, A. Petrone, T. Henderson, D. Ranasinghe, V. G. Zakrzewski, J. Gao, N. Rega, G. Zheng, W. Liang, M. Hada, M. Ehara, K. Toyota, R. Fukuda, J. Hasegawa, M. Ishida, T. Nakajima, Y. Honda, O. Kitao, H. Nakai, T. Vreven, K. Throssell, J. A. Montgomery, Jr., J. E. Peralta, F. Ogliaro, M. Bearpark, J. J. Heyd, E. Brothers, K. N. Kudin, V. N. Staroverov, T. Keith, R. Kobayashi, J. Normand, K. Raghavachari, A. Rendell, J. C. Burant, S. S. Iyengar, J. Tomasi, M. Cossi, J. M. Millam, M. Klene, C. Adamo, R. Cammi, J. W. Ochterski, R. L. Martin, K. Morokuma, O. Farkas, J. B. Foresman and D. J. Fox, Gaussian 09, Revision C.01, Gaussian Inc, Wallingford CT, 2016.

60 J. Tong, X. Xiao, X. Liang, N. von Solms, F. Huo, H. He and $\mathrm{S}$. Zhang, Insights into the solvation and dynamic behaviors of a lithium salt in organic- and ionic liquid-based electrolytes, Phys. Chem. Chem. Phys., 2019, 21, 19216-19225.

61 L. T. Costa, B. Sun, F. Jeschull and D. Brandell, Polymerionic liquid ternary systems for Li-battery electrolytes: Molecular dynamics studies of LiTFSI in a EMIm-TFSI and PEO blend, J. Chem. Phys., 2015, 143, 024904.

62 D. Bedrov, J.-P. Piquemal, O. Borodin, A. D. MacKerell, B. Roux and C. Schröder, Molecular Dynamics Simulations of Ionic Liquids and Electrolytes Using Polarizable Force Fields, Chem. Rev., 2019, 119, 7940-7995.
63 H. J. C. Berendsen, J. R. Grigera and T. P. Straatsma, The missing term in effective pair potentials, J. Phys. Chem., 1987, 91(24), 6269-6271.

64 L. Martínez, R. Andrade, E. G. Birgin and J. M. Martínez, PACKMOL: a package for building initial configurations for molecular dynamics simulations, J. Comput. Chem., 2009, 30, 2157-2164.

65 G. Bussi, D. Donadio and M. Parrinello, Canonical sampling through velocity rescaling, J. Chem. Phys., 2007, 126, 014101.

66 H. J. C. Berendsen, J. P. M. Postma, W. F. van Gunsteren, A. DiNola and J. R. Haak, Molecular dynamics with coupling to an external bath, J. Chem. Phys., 1984, 81, 3684-3690.

67 J.-H. Choi, H. Lee, H. R. Choi and M. Cho, Graph Theory and Ion and Molecular Aggregation in Aqueous Solutions, Annu. Rev. Phys. Chem., 2018, 69, 125-149.

68 M. G. Del Pópolo and G. A. Voth, On the Structure and Dynamics of Ionic Liquids, J. Phys. Chem. B, 2004, 108, 1744-1752.

69 M. P. Allen and D. J. Tildesley, Computer Simulation of Liquids, Clarendon Press, 1989.

70 M. Chintapalli, K. Timachova, K. R. Olson, S. J. Mecham, D. Devaux, J. M. DeSimone and N. P. Balsara, Relationship between Conductivity, Ion Diffusion, and Transference Number in Perfluoropolyether Electrolytes, Macromolecules, 2016, 49, 3508-3515.

71 J. Newman and K. E. Thomas-Alyea, Electrochemical Systems, Wiley, 3rd edn, 2004.

72 R. Kubo, Statistical-Mechanical Theory of Irreversible Processes. I. General Theory and Simple Applications to Magnetic and Conduction Problems, J. Phys. Soc. Jpn., 1957, 12, 570-586.

73 M. French, S. Hamel and R. Redmer, Dynamical Screening and Ionic Conductivity in Water from Ab Initio Simulations, Phys. Rev. Lett., 2011, 107, 185901.

74 T. D. N. Reddy and B. S. Mallik, Protic ammonium carboxylate ionic liquids: insight into structure, dynamics and thermophysical properties by alkyl group functionalization, Phys. Chem. Chem. Phys., 2017, 19, 10358-10370.

75 C. Rey-Castro and L. F. Vega, Transport Properties of the Ionic Liquid 1-Ethyl-3-Methylimidazolium Chloride from Equilibrium Molecular Dynamics Simulation. The Effect of Temperature, J. Phys. Chem. B, 2006, 110, 14426-14435.

76 J. Fiates, Y. Zhang, L. F. M. Franco, E. J. Maginn and G. Doubek, Impact of anion shape on $\mathrm{Li}+$ solvation and on transport properties for lithium-air batteries: a molecular dynamics study, Phys. Chem. Chem. Phys., 2020, 22, 15842-15852.

77 M. H. Kowsari, S. Alavi, M. Ashrafizaadeh and B. Najafi, Molecular dynamics simulation of imidazolium-based ionic liquids. II. Transport coefficients, J. Chem. Phys., 2009, 130, 014703.

78 M. H. Kowsari, S. Alavi, B. Najafi, K. Gholizadeh, E. Dehghanpisheh and F. Ranjbar, Molecular dynamics simulations of the structure and transport properties of tetra-butylphosphonium amino acid ionic liquids, Phys. Chem. Chem. Phys., 2011, 13, 8826-8837. 
79 A. Mondal and S. Balasubramanian, A Molecular Dynamics Study of Collective Transport Properties of ImidazoliumBased Room-Temperature Ionic Liquids, J. Chem. Eng. Data, 2014, 59, 3061-3068.

80 Q. Li, J. Chen, L. Fan, X. Kong and Y. Lu, Progress in electrolytes for rechargeable Li-based batteries and beyond, Green Energy Environ., 2016, 1, 18-42.

81 M. Winter and R. J. Brodd, What Are Batteries, Fuel Cells, and Supercapacitors?, Chem. Rev., 2004, 104, 4245-4270.

82 A. Luzar and D. Chandler, Effect of Environment on Hydrogen Bond Dynamics in Liquid Water, Phys. Rev. Lett., 1996, 76, 928-931.

83 A. Luzar and D. Chandler, Hydrogen-bond kinetics in liquid water, Nature, 1996, 379, 55-57.

84 A. Luzar, Resolving the hydrogen bond dynamics conundrum, J. Chem. Phys., 2000, 113, 10663-10675.

85 A. Chandra, Effects of Ion Atmosphere on Hydrogen-Bond Dynamics in Aqueous Electrolyte Solutions, Phys. Rev. Lett., 2000, 85, 768-771.

86 S. Chowdhuri and A. Chandra, Molecular dynamics simulations of aqueous $\mathrm{NaCl}$ and $\mathrm{KCl}$ solutions: Effects of ion concentration on the single-particle, pair, and collective dynamical properties of ions and water molecules, J. Chem. Phys., 2001, 115, 3732-3741.

87 B. S. Mallik and A. Chandra, Hydrogen bond and residence dynamics of ion-water and water-water pairs in supercritical aqueous ionic solutions: Dependence on ion size and density, J. Chem. Phys., 2006, 125, 234502.

88 S. Gehrke, M. von Domaros, R. Clark, O. Hollóczki, M. Brehm, T. Welton, A. Luzar and B. Kirchner, Structure and lifetimes in ionic liquids and their mixtures, Faraday Discuss., 2017, 206, 219-245.

89 S. Balasubramanian, S. Pal and B. Bagchi, Hydrogen-Bond Dynamics near a Micellar Surface: Origin of the Universal Slow Relaxation at Complex Aqueous Interfaces, Phys. Rev. Lett., 2002, 89, 115505.
90 N. Rawat and P. Biswas, Hydrogen Bond Dynamics in Intrinsically Disordered Proteins, J. Phys. Chem. B, 2014, 118, 3018-3025.

91 R. Shi and Y. Wang, Ion-Cage Interpretation for the Structural and Dynamic Changes of Ionic Liquids under an External Electric Field, J. Phys. Chem. B, 2013, 117, 5102-5112.

92 C. Hardacre, J. D. Holbrey, S. E. J. McMath, D. T. Bowron and A. K. Soper, Structure of molten 1,3dimethylimidazolium chloride using neutron diffraction, J. Chem. Phys., 2002, 118, 273-278.

93 Y. Zhang and E. J. Maginn, Direct Correlation between Ionic Liquid Transport Properties and Ion Pair Lifetimes: A Molecular Dynamics Study, J. Phys. Chem. Lett., 2015, 6, 700-705.

94 W. Zhao, F. Leroy, B. Heggen, S. Zahn, B. Kirchner, S. Balasubramanian and F. Müller-Plathe, Are There Stable Ion-Pairs in Room-Temperature Ionic Liquids? Molecular Dynamics Simulations of 1- $n$-Butyl-3-methylimidazolium Hexafluorophosphate, J. Am. Chem. Soc., 2009, 131, 15825-15833.

95 M. Kohagen, M. Brehm, J. Thar, W. Zhao, F. Müller-Plathe and B. Kirchner, Performance of Quantum Chemically Derived Charges and Persistence of Ion Cages in Ionic Liquids. A Molecular Dynamics Simulations Study of 1- $n$ Butyl-3-methylimidazolium Bromide, J. Phys. Chem. B, 2011, 115, 693-702.

96 D. van der Spoel, P. J. van Maaren, P. Larsson and N. Tîmneanu, Thermodynamics of Hydrogen Bonding in Hydrophilic and Hydrophobic Media, J. Phys. Chem. B, 2006, 110, 4393-4398.

97 G. Williams, D. C. Watts, S. B. Dev and A. M. North, Further considerations of non symmetrical dielectric relaxation behaviour arising from a simple empirical decay function, Trans. Faraday Soc., 1971, 67, 1323-1335.

98 M. McEldrew, Z. A. H. Goodwin, S. Bi, A. A. Kornyshev and M. Z. Bazant, Ion Clusters and Networks in Water-in-Salt Electrolytes, J. Electrochem. Soc., 2021, 168, 050514. 